\title{
LA EVOLUCION DE LA POBLACION MUNICIPAL EN EL PERIODO 1970-1975
}

$311(46) \times 1970 / 1975$ »

\author{
por \\ Ignacio Ballester Ros
}

SUMARIO: I. INTRODUCCION.-II. EL NUMERO DE MUNICIPIOS.1II. EVOlUCION DE LA POBlaCiON: 1. EN CONJUNTo. 2. Por Provincias. 3. Por capitales de Provincia.-IV. DISTRIBUCION DE LA POBLACION POR TAMAÑO DE LOS MUNICIPIOS.

\section{INTRODUCCION}

Por Real Decreto 30/1977, de 4 de enero, han sido declaradas oficiales las cifras de población de derecho y de hecho resultantes de la renovación del Padrón Municipal de Habitantes en todos los Municipios del país con referencia al 31 de diciembre de 1975.

$\mathrm{Y}$, en virtud de lo previsto en el mismo Decreto, el Instituto Nacional de Estadística ha editado una monografía en la que se consigna la población de derecho y de hecho de cada uno de los Municipios españoles.

Además, atendiendo a las recomendaciones internacionales en la materia, el propio Instituto está realizando una explotación estadística, mediante una muestra al 25 por 100, de la información contenida en los mencionados Padrones, relativa a las características personales, familiares y sociales de los españoles.

Debe resaltarse el hecho de que, por vez primera, se hayan declarado oficiales las cifras del Padrón Municipal de Habitantes. El período de diez años que transcurre entre cada dos censos generales de población es muy dilatado, cuando se producen cambios estructurales en la manera de ser y de asentar la población en cortos períodos de tiempo, y, por ello, ha resultado aconsejable aprovechar 
la renovación quinquenal del Padrón en un período intercensal, para otorgar a sus cifras este carácter oficial a todos los efectos administrativos.

Aunque de la declaración de oficiales han quedado excluidas, de momento, las cifras correspondientes a los Municipios de Madrid y Barcelona, y a otros cinco Municipios más pequeños, ello no ha de ser óbice para que realicemos un somero análisis de la evolución de la población española, a escala local, durante el repetido quinquenio, basándonos en la primera monografía citada, por el extraordinario interés que reviste.

\section{EL NUMERO DE MUNICIPIOS}

En el año 1970 se censaron 8.655 Municipios, y en 1975 se ha registrado la existencia de 8.194 . Se aprecia una disminución, en el quinquenio, de 461 Municipios, lo que equivale al 5 por 100 del total.

Su distribución, según el número de sus habitantes, en intervalos que pueden corresponderse con las zonas de residencia -rural, intermedia y urbana-, es:

\begin{tabular}{|c|c|c|}
\hline & 1970 & 1975 \\
\hline . & \multicolumn{2}{|c|}{ Cifras absolutas } \\
\hline De hasta 2.000 habitantes $\ldots \ldots \ldots c c$ & 6.383 & 5.983 \\
\hline 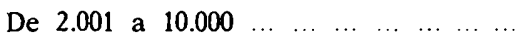 & 1.784 & 1.688 \\
\hline 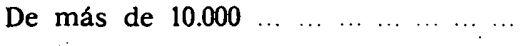 & 488 & 523 \\
\hline . & \multicolumn{2}{|c|}{ Porcentajes } \\
\hline De hasta 2.000 habitantes $\ldots \ldots \ldots c$ & 73,8 & 73,0 \\
\hline 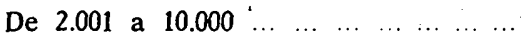 & $\because 20,6$ & 20,6 \\
\hline 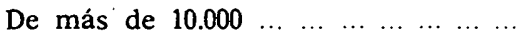 & 5,6 & 6,4 \\
\hline
\end{tabular}

Se advierte una disminución en el número de Municipios de las dos primeras zonas, que se corresponde con un incremento en los de la zona urbaná, lo que, en definitiva, apunta hacia una continuidad e incluso intensificación del proceso de concentración de la población en los Municipios de mayor número de habitantes. 
El detalle, por Provincias, es éste:

\begin{tabular}{|c|c|c|c|c|c|c|}
\hline \multicolumn{7}{|c|}{ Número de Municipios } \\
\hline \multicolumn{2}{|l|}{ Provincias } & 1970 & Total & $\begin{array}{l}\text { De hasta } \\
2.000 \text { hab. }\end{array}$ & $\begin{array}{l}\text { De } 2.001 \\
\text { a } 10.000\end{array}$ & $\begin{array}{l}\text { De más } \\
\text { de } 10.000\end{array}$ \\
\hline $\begin{array}{l}\text { Alava } \ldots \ldots \ldots \\
.\end{array}$ & $\ldots$ & 63 & 59 & 53 & 4 & 2 \\
\hline Albacete $\ldots \ldots \ldots$ & $\ldots$ & 86 & 86 & 46 & 35 & 5 \\
\hline Alicante $\ldots \ldots$ & $\ldots$ & 140 & 138 & 78 & 37 & 23 \\
\hline Almería $\ldots \ldots \ldots$ & $\ldots$ & 103 & 103 & 67 & 28 & 8 \\
\hline Avila $\ldots \ldots \ldots$ & $\ldots$ & 268 & 262 & 245 & 16 & 1 \\
\hline Badajoz $\ldots \ldots \ldots$ & $\ldots$ & 162 & 162 & 80 & 72 & 10 \\
\hline Baleares $\ldots \ldots$. & $\ldots$ & 65 & 65 & 14 & 39 & 12 \\
\hline Barcelona ... . & $\cdots$ & 308 & 308 & 169 & 92 & 47 \\
\hline $\begin{array}{llll}\text { Burgos } & \ldots & \ldots & \text {. }\end{array}$ & $\ldots$ & 485 & 439 & 424 & 12 & 3 \\
\hline $\begin{array}{llll}\text { Cáceres } & \ldots & \ldots\end{array}$ & $\ldots$ & 219 & 218 & 171 & 43 & 4 \\
\hline Cádiz $\ldots \ldots \ldots \ldots$ & $\ldots$ & 42 & 42 & 3 & 18 & 21 \\
\hline Castellón $\ldots$. & $\ldots$ & 142 & 140 & 110 & 21 & 9 \\
\hline Ciudad Real. & $\ldots$ & 98 & 98 & 49 & 37 & 12 \\
\hline Córdoba $\ldots \ldots \ldots$ & $\ldots$ & 75 & 75 & 17 & 44 & 14 \\
\hline Coruña (La) ... & $\ldots$ & 93 & 93 & 2 & 69 & 22 \\
\hline $\begin{array}{lllll}\text { Cuenca } & \ldots & \ldots & \end{array}$ & $\ldots$ & 280 & 242 & 221 & 20 & 1 \\
\hline Gerona & $\ldots$ & 235 & 223 & 187 & 27 & 9 \\
\hline Granada $\ldots \ldots \ldots$ & $\ldots$ & 194 & 169 & 93 & 66 & 10 \\
\hline Guadalajara... . & $\ldots$ & 335 & 295 & 288 & 6 & 1 \\
\hline Guipúzcoa $\ldots .$. & $\ldots$ & 81 & 81 & 39 & 21 & 21 \\
\hline $\begin{array}{llll}\text { Huelva } & \ldots & \ldots\end{array}$ & $\ldots$ & 79 & 79 & 31 & 41 & 7 \\
\hline $\begin{array}{llll}\text { Huesca } & \ldots & \ldots\end{array}$ & $\ldots$ & 254 & 209 & 195 & 9 & 5 \\
\hline $\begin{array}{llllll}\text { Jaén } & \ldots & \ldots & \ldots\end{array}$ & $\ldots$ & 101 & 96 & 20 & 60 & 16 \\
\hline $\begin{array}{lllll}\text { León } & \ldots & \ldots & \ldots\end{array}$ & $\ldots$ & 230 & 222 & 172 & 45 & 5 \\
\hline $\begin{array}{lllll}\text { Lérida } & \ldots & \ldots & \text {. }\end{array}$ & $\ldots$ & 255 & 230 & 198 & 29 & 3 \\
\hline Logroño $\ldots \ldots \ldots$ & $\ldots$ & 183 & 176 & 158 & 15 & 3 \\
\hline $\begin{array}{lllll}\text { Lugo } & \ldots & \ldots & \ldots\end{array}$ & $\ldots$ & 66 & 66 & 6 & 54 & 6 \\
\hline Madrid $\ldots \ldots \ldots$. & $\ldots$ & 183 & 179 & 126 & 34 & 19 \\
\hline $\begin{array}{llll}\text { Málaga } & \ldots & \ldots\end{array}$ & $\ldots$ & 100 & 99 & 43 & 41 & 15 \\
\hline $\begin{array}{llll}\text { Murcia } & \ldots & \ldots & \end{array}$ & $\ldots$ & 43 & 43 & 5 & 18 & 20 \\
\hline $\begin{array}{cccc}\text { Navarra } & \ldots & \ldots\end{array}$ & $\ldots$ & 265 & 264 & 216 & 44 & 4 \\
\hline $\begin{array}{lllll}\text { Orense } & \ldots & \ldots & .\end{array}$ & $\ldots$ & 92 & 92 & 13 & 78 & 1 \\
\hline $\begin{array}{llll}\text { Oviedo } & \ldots & \ldots\end{array}$ & $\ldots$ & 78 & 78 & 19 & 36 & 23 \\
\hline Palencia & $\ldots$ & 246 & 203 & 189 & 13 & 1. \\
\hline Palmas (Las). & $\ldots$ & 34 & 34 & 4 & 18 & 12 \\
\hline Pontevedra .... & $\ldots$ & 61 & 61 & 1 & 39 & 21 \\
\hline Salamanca .... & $\ldots$ & 382 & 359 & 348 & 8 & 3 \\
\hline S. C. Tenerife . & $\ldots$ & 53 & 53 & 5 & 34 & 14 \\
\hline Santander ... & $\ldots$ & 102 & 102 & 54 & 40 & 8 \\
\hline $\begin{array}{llll}\text { Segovia } & \ldots & \ldots & \end{array}$ & $\ldots$ & 234 . & 215 & 206 & 8 & 1 \\
\hline $\begin{array}{lllll}\text { Sevilla } & \ldots & \ldots & \text {. }\end{array}$ & $\ldots$ & 102 & 102 & 14 & 64 & 24 \\
\hline $\begin{array}{ccccc}\text { Soria } & \ldots & \ldots & \ldots\end{array}$ & $\ldots$ & 219 & 185 & 178 & 6 & 1 \\
\hline Tarragona $\ldots .$. & $\ldots$ & 178 & 177 & 140 & 30 & 7 \\
\hline Teruel $\ldots$ & $\ldots$ & 268 & 234 & 222 & 10 & 2 \\
\hline $\begin{array}{llll}\text { Toledo } & \ldots & \ldots \\
\text {. }\end{array}$ & $\ldots$ & 205 & 204 & 143 & 59 & 2 \\
\hline Valencia $\ldots \ldots .$. & $\ldots$ & 263 & 263 & 148 & 78 & 37 \\
\hline Valladolid $\ldots .$. & $\ldots$ & 233 & 229 & 214 & 13 & 2 \\
\hline $\begin{array}{lllll} & \text { Vizcaya } & \ldots & \ldots\end{array}$ & $\ldots$ & 97 & 96 & 52 & 26 & 18 \\
\hline Zamora $\ldots \ldots$. & $\ldots$ & 275 & 252 & 245 & 5 & 2 \\
\hline Zaragoza ... ... & $\ldots$ & 298 & 292 & 262 & 26 & 4 \\
\hline $\begin{array}{ccccc}\text { Ceuta } & \ldots & \ldots & \ldots & \end{array}$ & $\ldots$ & 1 & 1 & & & 1 \\
\hline $\begin{array}{ccccl}\text { Melilla } & \ldots & \ldots & .\end{array}$ & $\ldots$ & 1 & 1 & & & 1 \\
\hline
\end{tabular}


Del examen de la tabla anterior se deduce, en primer lugar, que la anotada disminución en el número de Municipios afecta básicamente a trece Provincias:

- Burgos, Palencia, Zamora, Salamanca, Segovia y Soria, en el Valle del Duero.

- Cuenca y Guadalajara, en la Submeseta Sur.

- Huesca, Lérida y Teruel, en el Valle del Ebro.

- Gerona, en el extremo nororiental.

- Granada, al Sur.

Esta disminución ha sido más limitada en León, Valladolid, Avila, Logroño, Zaragoza, Madrid y Jaén, y se ha reducido a uno o dos Municipios en Vizcaya, Navarra, Cáceres, Toledo, Tarragona, Castellón y Alicante.

A pesar de la reducción operada en el número de Municipios, puede afirmarse que, en esencia, la estructura de los Municipios españoles sigue siendo la misma.

Se caracteriza, de una parte, por un predominio numérico de pequeños Municipios en las Regiones históricas de León, ambas Castillas, Aragón, Cataluña y Valencia, que registra menor intensidad en las Vascongadas y en las Provincias de Santander, Albacete y Ciudad Real, Granada, Málaga y Almería. Y, de otra, por su escaso número en Galicia y Asturias -compatible con el gran número de entidades colectivas y singulares en que se ubica la población sobre el territorio-, Baleares y Canarias y en las cinco Provincias andaluzas restantes.

Una vez más, hay que subrayar la desigualdad demográfica de los Municipios españoles, que repercute de manera desfavorable en el distinto grado de disfrute de los servicios públicos y comunitarios por parte de los españoles en ellos residentes.

\section{EVOLUCION DE LA POBLACION}

\section{EN CONJUNTO}

Sin tener en cuenta la población de los Municipios de Madrid y Barcelona, que en el censo de 1970 representaban, en conjunto, 4.891.215 habitantes y de los otros cinco Municipios más pequeños, 
con 22.140, a que se ha aludido en la Introducción, la población de hecho censada en 1970 se elevó a 29.042 .694 habitantes, y en el Padrón de 1975 se cifró en 31.048.151, lo que representa un incremento, en el quinquenio, de 2.005 .457 habitantes, a un ritmo de 400.000 anuales.

Por tamaño de los Municipios, la distribución de la población es:

\begin{tabular}{|c|c|c|}
\hline & 1970 & 1975 \\
\hline & \multicolumn{2}{|c|}{ Cifras absolutas } \\
\hline De hasta 2.000 habitantes $\ldots \ldots \ldots$ & 3.734 .079 & 3.329 .819 \\
\hline 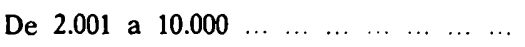 & 7.623 .861 & 7.600 .222 \\
\hline 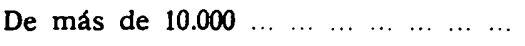 & 17.684 .754 & 20.118 .110 \\
\hline & \multicolumn{2}{|c|}{ Porcentajes } \\
\hline De hasta 2.000 habitantes $\ldots \begin{array}{llll}\ldots & \ldots & \ldots & \ldots\end{array}$ & 12,9 & 10,7 \\
\hline 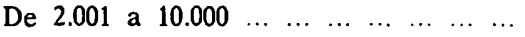 & 26,3 & 24,5 \\
\hline 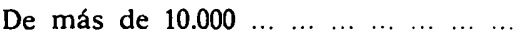 & 60,8 & 64,8 \\
\hline
\end{tabular}

El proceso de concentración de la población en los grandes Municipios se pone de manifiesto de manera muy acusada en las cifras anteriores. En tan sólo cinco años, un 4 por 100 de la población ha pasado a residir en Municipios mayores de 10.000 habitantes, donde se ubican ya dos de cada tres españoles; uno de cada diez habitantes reside en Municipios pequeños, y uno de cada cuatro, en los de la zona intermedia.

\section{POR Provincias}

En la siguiente tabla se mide la evolución de la población de hecho por Provincias, en el período de referencia, sin tener en cuenta, tanto en 1970 como en 1975, de las cifras de habitantes de los Municipios de Madrid y Barcelona y de los otros cinco a que se alude en el apartado anterior. 


\begin{tabular}{|c|c|c|c|c|}
\hline Provincias & $\begin{array}{c}\text { a) } \\
1970\end{array}$ & $\begin{array}{l}\text { b) } \\
1975\end{array}$ & c) & $\frac{\text { c) }}{\text { a) }} 100$ \\
\hline lava & 204.323 & 238.223 & 33.900 & 16,5 \\
\hline ......... & 335.026 & 331.390 & 3.636 & $-1,1$ \\
\hline $\begin{array}{lllllll}\text { Alicante } & \ldots & \ldots & \ldots & \ldots & \ldots & \ldots\end{array}$ & 920.105 & 1.060 .601 & 140.496 & 15,3 \\
\hline $\begin{array}{llllllll}\text { Almería } & \ldots & \ldots & \ldots & \ldots & \ldots & \ldots\end{array}$ & 375.004 & 386.776 & 11.772 & 3,1 \\
\hline $\begin{array}{lllllllll}\text { Avila } & \ldots & \ldots & \ldots & \ldots & \ldots & \ldots & \ldots\end{array}$ & 203.798 & 187.725 & -16.073 & \\
\hline $\begin{array}{ccccccc}\text { Badajoz } & \ldots & \ldots & \ldots & \ldots & \ldots & \ldots\end{array}$ & 687.599 & 640.850 & -46.749 & $-6,8$ \\
\hline $\begin{array}{lllllll}\text { Baleares } & \ldots & \ldots & \ldots & \ldots & \ldots & \ldots\end{array}$ & 558.287 & 633.016 & 74.729 & 13,4 \\
\hline $\begin{array}{llllll}\text { Barcelona } & (1) & \ldots & \ldots & \ldots & \ldots\end{array}$ & 2.184 .052 & 2.632 & 448.553 & 20,5 \\
\hline $\begin{array}{lllllll}\text { Burgos } & \ldots & \ldots & \ldots & \ldots & \ldots & \ldots\end{array}$ & 358.075 & 349.347 & -8.728 & $-2,4$ \\
\hline $\begin{array}{llllllll}\text { Cáceres } & \ldots & \ldots & \ldots & \ldots & \ldots & \ldots\end{array}$ & 457.777 & 425.667 & -32.110 & $-7,0$ \\
\hline $\begin{array}{llllll}. . & \ldots & \ldots & \ldots & . . & \\
\end{array}$ & 88. & 95 & 66 & 7,6 \\
\hline $\begin{array}{lllll}. & \ldots & \ldots & \ldots & . \\
\end{array}$ & 385 & 411.129 & 25.306 & 6,6 \\
\hline Ciudad Real $\ldots \ldots \ldots \ldots$ & 507. & 481.212 & -26.438 & $-5,2$ \\
\hline . ...... & 724 & 71 & -7 & $-1,0$ \\
\hline $\begin{array}{lllllll}\text { Coruña }(\mathrm{La}) & \ldots & \ldots & \ldots & \ldots & \end{array}$ & 1.004 & 1.042 & 38.692 & 3,9 \\
\hline $\begin{array}{llllllll}\text { Cuenca } & \ldots & \ldots & \ldots & \ldots & \ldots & \ldots & \ldots\end{array}$ & 247 & 222.306 & -24.852 & $-10,1$ \\
\hline 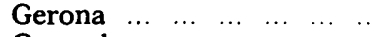 & 41 & 44 & 27.593 & 6,7 \\
\hline $\begin{array}{lllll}\ldots & \ldots & \ldots & . .\end{array}$ & 733 & 736 & 2.670 & 0,4 \\
\hline $\begin{array}{cccccc}G u a d a l a j a r a & \ldots & \ldots & \ldots & \ldots & \ldots\end{array}$ & 147. & 139 & -8.208 & $-5,6$ \\
\hline $\begin{array}{ccccccc}\mathbf{z c o a} & \ldots & \ldots & \ldots & \ldots & \ldots & \ldots\end{array}$ & 631 & 682 & 51. & 8,2 \\
\hline $\begin{array}{llllllll}\text { Huelva } & \ldots & \ldots & \ldots & \ldots & \ldots & \ldots & \ldots\end{array}$ & 397. & 400 & 21 & 0,6 \\
\hline $\begin{array}{lllllll} & \ldots & \ldots & \ldots & \ldots & \ldots & \ldots\end{array}$ & 222. & 216 & -5.893 & $-2,7$ \\
\hline $\begin{array}{lllllll} & \ldots & \ldots & \ldots & \ldots & \ldots & \end{array}$ & 661 & 645 & -15.622 & $-2,4$ \\
\hline $\begin{array}{ccccc}\ldots & \ldots & \ldots & \ldots & \ldots \\
\end{array}$ & 548 & 526 & -22.225 & $-4,1$ \\
\hline $\begin{array}{llllll}\ldots & \ldots & \ldots & \ldots & \ldots & . .\end{array}$ & 347 & 349 & 2.218 & 0,6 \\
\hline $\begin{array}{lllllll} & \ldots & \ldots & \ldots & \ldots & \ldots & \ldots\end{array}$ & 235 & 240 & 5.023 & 2,1 \\
\hline $\begin{array}{llllll}\ldots & \ldots & \ldots & \ldots & \ldots & \\
\end{array}$ & 399 & 390 & -9.172 & $-2,3$ \\
\hline$\cdots \ldots \ldots$ & 646 & 1.092 & 446 & 69,0 \\
\hline $\begin{array}{llllllll} & \ldots & \ldots & \ldots & \ldots & \ldots & \ldots & \ldots \\
\end{array}$ & 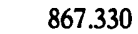 & & 51 & 6,0 \\
\hline $\begin{array}{llllll}. & \ldots & \ldots & \ldots & \ldots & \ldots\end{array}$ & 832 & 88 & 51 & 6,2 \\
\hline ....... & 464 & 483 & 19. & A \\
\hline ....... & 408 & 404 & -3 & $-0,8$ \\
\hline $\begin{array}{llllllll} & \ldots & \ldots & \ldots & \ldots & \ldots & \ldots & \ldots\end{array}$ & 1.04 & 1.09 & & 5,1 \\
\hline 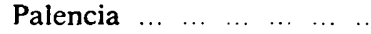 & 198 & 186 & -12 & 6,1 \\
\hline$\ldots \ldots \ldots$ & & & 127 & 22,0 \\
\hline $\begin{array}{llllll}\text { vedra } & \ldots & \ldots & \ldots & \ldots & \ldots\end{array}$ & 750 & 825 & & 10,0 \\
\hline Salamanca & 371 & 349 & -21 & 5,9 \\
\hline ta Cruz de Tenerife .. & 590 & 686 & 96.444 & 16,3 \\
\hline $\begin{array}{lllllll}\text { nder } & \ldots & \ldots & \ldots & \ldots & \ldots & \ldots\end{array}$ & 467. & & & 10 \\
\hline$\ldots \ldots \ldots$ & 162 & 0 & -11 & \\
\hline$\ldots \ldots$ & 1.327 .190 & 1.375 & 48 & 3,6 \\
\hline $\begin{array}{llllll}\ldots & \ldots & \ldots & \ldots & \ldots \\
\end{array}$ & 114.956 & 103 & -11 & 9,6 \\
\hline gona & 431 & 48 & 52 & 12,2 \\
\hline 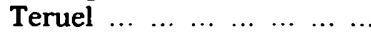 & 170.284 & 155.449 & -14.835 & $-8,7$ \\
\hline $\begin{array}{lllll}\ldots & \ldots & \ldots & . .\end{array}$ & 468. & 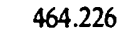 & -4.699 & $-1,0$ \\
\hline & 1.767 & 1.939 & 172.161 & 9,7 \\
\hline Valladolid & 412.572 & 450.670 & 38.098 & 9,2 \\
\hline Vizcaya (5) & 1.042 .277 & 1.150 & 108.316 & 10,4 \\
\hline ora $\ldots$ & 251.934 & 230 & -21.147 & $-8,4$ \\
\hline $\begin{array}{llllllll}\text { Zaragoza } & \ldots & \ldots & \ldots & \ldots & \ldots & . .\end{array}$ & 760.186 & 802.031 & 41.845 & 5,5 \\
\hline $\begin{array}{llllll}\ldots & \ldots & \ldots & \ldots & \ldots & . .\end{array}$ & 67.187 & 67.077 & 110 & $-0,2$ \\
\hline & 65.271 & 59.616 & -5.655 & $-8,7$ \\
\hline
\end{tabular}


En el conjunto nacional se ha registrado un incremento de la población del 6,8 por 100 ; pero la tabla anterior registra una fuerte dispersión de los valores representativos de la evolución de cada Provincia alrededor de este promedio nacional de aumento de la población española en el quinquenio que analizamos.

De una parte, figuran un conjunto de 20 Provincias en las que se ha dado un incremento, en cifras absolutas, de 2.337.905 habitantes; y, de otra, otro conjunto de 21 Provincias y, además, Ceuta y Melilla, que presentan una disminución conjunta de 332.448 habitantes.

Por encima del promedio nacional de incremento destacan 14 Provincias, a las que podemos añadir otras dos - Castellón y Geronaque tienen un valor muy próximo, y que configuran las siguientes zonas que pueden estimarse las más prósperas en el orden demográfico, y paralelamente en el económico, al constituir fuertes focos de atracción de la población:

- Pontevedra.

- Vascongadas.

- Madrid y Valladolid.

- Litoral mediterráneo, con Gerona, Barcelona, Tarragona, Castellón, Valencia, Alicante y Baleares.

- Cádiz.

- Canarias.

Es de señalar el singular incremento registrado por la Provincia de Madrid, que, sin tener en cuenta la capital, se eleva a 450.000 habitantes, equivalente al 69 por 100 de la población de 1970. Hay que atribuir este aumento al de los Municipios que constituyen la zona de influencia inmediata a la Capital, con la zona industrial y los municipios-dormitorio. Alcalá de Henares, Alcorcón, Getafe y Leganés superan los 100.000 habitantes; Alcobendas y Móstoles rebasan los 50.000, y por encima de los 20.000 se sitúan Coslada, Pozuelo de Alarcón, Parla, San Sebastián de los Reyes, Torrejón de Ardoz y, más alejado, Aranjuez.

La Provincia de Barcelona - sin la Capital- ofrece un aumento similar en cifras absolutas; pero que representa el 20 por 100 de la población censal. Debe atribuirse este aumento a la comarca de Barcelona. Badalona, Hospitalet, Sabadell, Santa Coloma de Grama- 
net y Tarrasa rebasan los 100.000 habitantes, y algunos de ellos con notable exceso; Cornellá, Manresa, Mataró, Prat de Llobregat y San Baudilio de Llobregat superan los 50.000, y superan los 20.000 habitantes 17 Municipios más.

En orden de importancia en razón del valor absoluto del aumento de población, superior a los 100.000 habitantes, figuran detrás de Madrid y Barcelona las Provincias de Valencia, Alicante, Las Palmas y Vizcaya. Las 13 Provincias restantes, con incrementos inferiores al promedio nacional, y en algunos casos - Granada, Huelva, Lérida - tan escasos que se pueden considerar como Provincias de población estacionaria, sumadas a las 21 en las que la población disminuye, forman un conjunto de 34 , demográficamente atrasadas. Dos de cada tres Provincias son deficitarias en habitantes y no pueden seguir el ritmo del incremento medio nacional.

En valores relativos, tras el espectacular aumento de la población en la Provincia de Madrid, Las Palmas supera a Barcelona, y por debajo de ellas se sitúan, con un aumento superior a la sexta parte de su respectiva población: Alava, Santa Cruz de Tenerife y Alicante; y con el 10 por 100 o más: Baleares, Tarragona, Pontevedra y Vizcaya. No llegan al 10 por 100 de incremento, aunque superen el promedio nacional, Valencia, Valladolid, Guipúzcoa y Cádiz.

Las 21 Provincias cuya población se ha visto reducida en el período estudiado se distribuyen, por Regiones históricas, en esta forma:

- Galicia: Lugo y Orense.

- León: León, Zamora, Salamanca y Palencia.

- Castilla la Vieja: Burgos, Soria, Segovia y Avila.

- Castilla la Nueva: Toledo, Ciudad Real, Cuenca y Guadalaja. ra, a la que puede unirse Albacete.

- Extremadura: Cáceres y Badajoz.

- Aragón: Huesca y Teruel.

- Andalucía: Córdoba y Jaén,

que constituyen una gran zona geográfica, prácticamente sin solu. ción de continuidad, que pudiéramos redistribuir en tres Regiones naturales:

- la Meseta: menos las Provincias de Madrid y Valladolid;

- el Valle del Ebro, con sólo Huesca y Teruel;

- Andalucía, con sólo Córdoba y Jaén. 


\section{Por capitales de Provincia}

Con la salvedad de Madrid y Barcelona, la población de las capitales de Provincia, que fue en 1970 de 7.199 .895 habitantes, se ha elevado en 1975 a 8.052 .934 , con un incremento absoluto de 853.039, lo que equivale al 11,8 por 100 del conjunto, que casi duplica la tasa de incremento provincial.

La población de las capitales equivale en 1975 al 25,9 por 100 de la población total, lo que supone decir que uno de cuatro habitantes reside en ellas.

En la tabla siguiente se presentan las cifras obtenidas en el censo de 1970 y en el Padrón de 1975, y los aumentos absolutos y relativos correspondientes.

\begin{tabular}{|c|c|c|c|c|}
\hline Capitales & $\begin{array}{c}\text { a) } \\
1970\end{array}$ & $\begin{array}{l}\text { b) } \\
1975\end{array}$ & c) & $\frac{\text { c) }}{\text { a) }} 100$ \\
\hline Vitoria & 136.873 & 170.870 & 33.997 & 24,8 \\
\hline $\begin{array}{lllllll}\text { Albacete } & \ldots & \ldots & \ldots & \ldots & \ldots & \ldots\end{array}$ & 93.233 & 101.815 & 8.582 & 9,2 \\
\hline $\begin{array}{lllllll}\text { Alicante } & \ldots & \ldots & \ldots & \ldots & \ldots & \ldots\end{array}$ & 184.716 & 219.553 & 34.837 & 18,9 \\
\hline $\begin{array}{ccccccc}\text { Almeria } & \ldots & \ldots & \ldots & \ldots & \ldots & \ldots\end{array}$ & 114.510 & 121.302 & 6.792 & 5,9 \\
\hline $\begin{array}{lllllllll}\text { Avila } & \ldots & \ldots & \ldots & \ldots & \ldots & \ldots & \ldots\end{array}$ & 30.983 & 34.263 & 3.280 & 10,6 \\
\hline 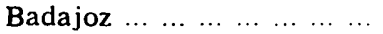 & 101.710 & 103.818 & 2.108 & 2,1 \\
\hline Palma de Mallorca $\ldots \ldots \ldots \ldots$ & 234.098 & 282.050 & 47.952 & 20,4 \\
\hline $\begin{array}{lllllllll}\text { Barcelona } & \ldots & \ldots & \ldots & \ldots & \ldots & \ldots\end{array}$ & 1.745 .142 & - & - & - \\
\hline $\begin{array}{lllllllll}\text { Burgos } & \ldots & \ldots & \ldots & \ldots & \ldots & \ldots & \ldots\end{array}$ & 119.915 & 134.682 & 14.767 & 12,3 \\
\hline $\begin{array}{lllllllll}\text { Cáceres } & \ldots & \ldots & \ldots & \ldots & \ldots & \ldots & \ldots\end{array}$ & 56.064 & 58.844 & 2.780 & 5,0 \\
\hline $\begin{array}{lllllllll}\text { Cádiz } & \ldots & \ldots & \ldots & \ldots & \ldots & \ldots & \ldots\end{array}$ & 135.743 & 142.242 & 6.499 & 4,8 \\
\hline $\begin{array}{llllllll}\text { Castellón } & \ldots & \ldots & \ldots & \ldots & \ldots & \ldots\end{array}$ & 93.968 & 109.882 & 15.914 & 16,9 \\
\hline $\begin{array}{lllllll}\text { Ciudad Real } & \ldots & \ldots & \ldots & \ldots & \ldots\end{array}$ & 41.708 & 45.247 & 3.539 & 8,5 \\
\hline $\begin{array}{llllllll}\text { Córdoba } & \ldots & \ldots & \ldots & \ldots & \ldots & \ldots\end{array}$ & 235.632 & 255.250 & 19.628 & 8,3 \\
\hline $\begin{array}{lllllll}\text { Coruña }(L a) & \ldots & \ldots & \ldots & \ldots & \ldots\end{array}$ & 189.654 & 207.269 & 17.615 & 9,3 \\
\hline $\begin{array}{cccccccc}\text { Cuenca } & \ldots & \ldots & \ldots & \ldots & \ldots & \ldots & \ldots\end{array}$ & 34.485 & 37.088 & 2.603 & 7,5 \\
\hline $\begin{array}{llllllll}\text { Gerona } & \ldots & \ldots & \ldots & \ldots & \ldots & \ldots & \ldots\end{array}$ & 50.338 & 75.600 & 25.262 & 50,2 \\
\hline $\begin{array}{llllllll}\text { Granada } & \ldots & \ldots & \ldots & \ldots & \ldots & \ldots\end{array}$ & 190.429 & 214.091 & 23.662 & 12,4 \\
\hline $\begin{array}{cccccc}\text { Guadalajara } & \ldots & \ldots & \ldots & \ldots & \ldots\end{array}$ & 31.917 & 45.162 & 13.245 & 41,5 \\
\hline $\begin{array}{llllll}\text { San Sebastián } & \ldots & \ldots & \ldots & \ldots\end{array}$ & 165.829 & 169.622 & 3.793 & 2,3 \\
\hline $\begin{array}{llllllll}\text { Huelva } & \ldots & \ldots & \ldots & \ldots & \ldots & \ldots & \ldots\end{array}$ & 96.689 & 111.238 & 14.549 & 15,0 \\
\hline $\begin{array}{cccccccc}\text { Huesca } & \ldots & \ldots & \ldots & \ldots & \ldots & \ldots & \ldots\end{array}$ & 33.185 & 37.610 & 4.425 & 13,3 \\
\hline $\begin{array}{lllllllll}\text { Jaén } & \ldots & \ldots & \ldots & \ldots & \ldots & \ldots & \ldots & \ldots\end{array}$ & 78.156 & 84.114 & 5.958 & 7,6 \\
\hline $\begin{array}{llllll} & \ldots & \ldots & \ldots & \ldots & \ldots\end{array}$ & 105.235 & 115.176 & 9.941 & 9,4 \\
\hline $\begin{array}{llllllll}\text { Lérida } & \ldots & \ldots & \ldots & \ldots & \ldots & \ldots & \ldots\end{array}$ & 90.884 & 102.599 & 11.715 & 12,9 \\
\hline $\begin{array}{llllllll}\text { Logroño } & \ldots & \ldots & \ldots & \ldots & \ldots & \ldots & \ldots\end{array}$ & 84.456 & 96.662 & 12.206 & 14,5 \\
\hline $\begin{array}{llllllll}\text { Lugo } & \ldots & \ldots & \ldots & \ldots & \ldots & \ldots & \ldots\end{array}$ & 63.830 & 68.163 & 4.333 & 6,8 \\
\hline $\begin{array}{llllllll}\text { Madrid } & \ldots & \ldots & \ldots & \ldots & \ldots & \ldots & \ldots\end{array}$ & 3.146 .071 & - & - & - \\
\hline $\begin{array}{llllllll}\text { Málaga } & \ldots & \ldots & \ldots & \ldots & \ldots & \ldots & \ldots\end{array}$ & 374.452 & 411.131 & 36.679 & 9,8 \\
\hline $\begin{array}{cccccccc}\text { Murcia } & \ldots & \ldots & \ldots & \ldots & \ldots & \ldots & \ldots\end{array}$ & 243.759 & 263.082 & 19.323 & 7,9 \\
\hline $\begin{array}{llllllll}\text { Pamplona } & \ldots & \ldots & \ldots & \ldots & \ldots & \ldots\end{array}$ & 147.168 & 165.277 & 18.109 & 12,3 \\
\hline $\begin{array}{lllllllll}\text { Orense } & \ldots & \ldots & \ldots & \ldots & \ldots & \ldots & \ldots\end{array}$ & 73.379 & 80.048 & 6.669 & 9,1 \\
\hline $\begin{array}{llllllll}\text { Oviedo } & \ldots & \ldots & \ldots & \ldots & \ldots & \ldots & \ldots\end{array}$ & 154.117 & 161.944 & 7.827 & 5,1 \\
\hline $\begin{array}{lllllll}\text { Palencia } & \ldots & \ldots & \ldots & \ldots & \ldots & \ldots\end{array}$ & 58.370 & 63.557 & 5.187 & 8,9 \\
\hline
\end{tabular}




\begin{tabular}{|c|c|c|c|c|}
\hline Capitales & $\begin{array}{c}\text { a) } \\
1970\end{array}$ & $\begin{array}{l}\text { b) } \\
1975\end{array}$ & c) & $\frac{\text { c) }}{\text { a) }} 100$ \\
\hline 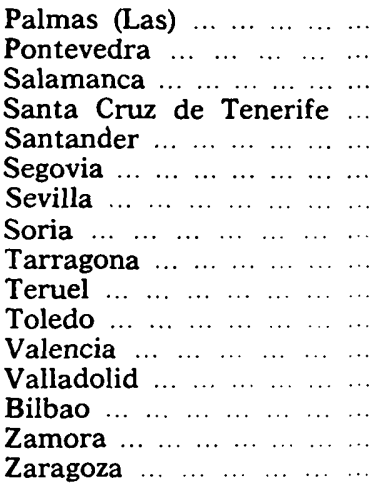 & $\begin{array}{r}287.038 \\
52.452 \\
125.220 \\
151.361 \\
149.704 \\
41.880 \\
548.072 \\
25.030 \\
78.238 \\
21.638 \\
44.382 \\
653.690 \\
236.341 \\
410.490 \\
49.029 \\
479.845\end{array}$ & $\begin{array}{r}348.776 \\
60.535 \\
133.288 \\
186.237 \\
164.999 \\
47.701 \\
590.235 \\
28.308 \\
101.619 \\
24.122 \\
52.988 \\
714.086 \\
287.230 \\
431.071 \\
52.180 \\
540.308\end{array}$ & $\begin{array}{r}61.738 \\
8.083 \\
8.068 \\
34.876 \\
15.295 \\
5.821 \\
42.163 \\
3.278 \\
23.381 \\
2.484 \\
8.606 \\
60.396 \\
50.889 \\
20.581 \\
3.151 \\
60.463\end{array}$ & $\begin{array}{r}21,5 \\
15,4 \\
6,4 \\
23,0 \\
10,2 \\
13,9 \\
7,7 \\
13,1 \\
29,9 \\
11,5 \\
19,4 \\
9,2 \\
21,5 \\
5,0 \\
6,4 \\
13,0\end{array}$ \\
\hline
\end{tabular}

Todas las capitales han aumentado su población en mayor o menor grado. Es el fiel reflejo del proceso de concentración urbana, fruto del movimiento migratorio interior hacia las zonas industriales y las grandes urbes.

Por el volumen absoluto de población las capitales -excluidas Madrid y Barcelona- pueden distribuirse, en 1975, de esta manera:

- Superan el medio millón de habitantes: Valencia, Sevilla y Zaragoza.

- Entre un cuarto de millón y medio millón se encuentran: Bilbao, Málaga, Las Palmas, Valladolid, Palma de Mallorca, Murcia y Córdoba.

- Con más de 100.000 habitantes, sin rebasar los 250.000 , se hallan 20 capitales.

- Las 18 capitales restantes tienen menos de 100.000 habitantes.

Entre las 22 capitales, cuyo porcentaje de aumento es superior al promedio de capitales, figuran Zaragoza, Valladolid, Las Palmas y Palma de Mallorca, pertenecientes a los dos primeros grupos; la mitad de las del tercer grupo y 8 que no superan los 100.000 .

Como se observa, ciudades de distintos niveles demográficos experimentan notables incrementos en el número de sus habitantes. En unos casos, como Gerona y Guadalajara, con los más elevados porcentajes, el fenómeno parece obedecer al establecimiento de polos de desarrollo industrial y a las anexiones a la capital de térmi- 
nos municipales colindantes. La industria y el turismo y la capitalidad de una zona agraria importante, o el desarrollo de los servicios, o la conjunción de ellas, son factores determinantes de los aumentos de población.

\section{DISTRIBUCION DE LA POBLACION POR TAMAÑO DE LOS MUNICIPIOS}

En el apartado III se ha presentado la población de los Municipios españoles agrupada en tres niveles correspondientes a las zonas de residencia rural, intermedia y urbana.

A fin de conocer la misma distribución por Provincias se han elaborado las dos tablas siguientes, con los valores absolutos y porcentajes correspondientes.

\begin{tabular}{|c|c|c|c|c|}
\hline \multirow[b]{2}{*}{ Provincias } & \multicolumn{4}{|c|}{ Población de los Municipios } \\
\hline & Total & $\begin{array}{l}\text { De hasta } \\
2.000 \text { hab. }\end{array}$ & $\begin{array}{l}\text { De } 2.001 \\
\text { a } 10.000\end{array}$ & $\begin{array}{l}\text { Mayores } \\
\text { de } 10.000\end{array}$ \\
\hline $\begin{array}{llllllll}\text { Alava } & \ldots & \ldots & \ldots & \ldots & \ldots & \ldots & \ldots\end{array}$ & 238.223 & 33.524 & 14.759 & 189.940 \\
\hline $\begin{array}{lllllll}\text { Albacete } & \ldots & \ldots & \ldots & \ldots & \ldots & \ldots\end{array}$ & 331.390 & 40.746 & 116.264 & 174.380 \\
\hline $\begin{array}{lllllll}\text { Alicante } & \ldots & \ldots & \ldots & \ldots & \ldots & \ldots\end{array}$ & 1.060 .601 & 54.651 & 185.206 & 820.744 \\
\hline 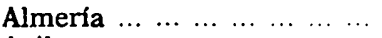 & 386.776 & 55.011 & 107.328 & 224.437 \\
\hline $\begin{array}{lllllllll}\text { Avila } & \ldots & \ldots & \ldots & \ldots & \ldots & \ldots & \ldots & \ldots\end{array}$ & 187.725 & 100.833 & 52.629 & 34.263 \\
\hline $\begin{array}{lllllll}\text { Badajoz } & \ldots & \ldots & \ldots & \ldots & \ldots & \ldots\end{array}$ & 640.850 & 82.969 & 289.379 & 268.502 \\
\hline $\begin{array}{lllllll}\text { Baleares } & \ldots & \ldots & \ldots & \ldots & \ldots & \ldots\end{array}$ & 633.016 & 12.251 & 466.589 & 154.176 \\
\hline $\begin{array}{cccccc}\text { Barcelona }(1) & \ldots & \ldots & \ldots & \ldots & \ldots\end{array}$ & 2.632 .605 & 110.660 & 410.911 & 2.111 .034 \\
\hline 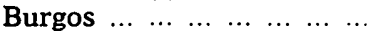 & 349.347 & 120.809 & 36.369 & 192.169 \\
\hline $\begin{array}{llllllll}\text { Cáceres } & \ldots & \ldots & \ldots & \ldots & \ldots & \ldots & \ldots\end{array}$ & 425.667 & 148.467 & 167.635 & 109.565 \\
\hline $\begin{array}{lllll}\cdots & \ldots & \ldots & \ldots & \ldots\end{array}$ & 952.328 & 2.320 & 85.135 & 864.873 \\
\hline $\begin{array}{lllll}\ldots & \ldots & \ldots & \ldots & \ldots\end{array}$ & 411.129 & 69.131 & 73.038 & 268.960 \\
\hline Ciudad Real $\ldots \ldots \ldots c c c$ & 481.212 & 47.962 & 171.232 & 262.018 \\
\hline $\begin{array}{lllllll}\text { Córdoba } & \ldots & \ldots & \ldots & \ldots & \ldots & \ldots\end{array}$ & 717.005 & 21.182 & 211.940 & 483.883 \\
\hline $\begin{array}{llllll}\text { Coruña }(\text { La) } & \ldots & \ldots & \ldots & \ldots & \ldots\end{array}$ & 1.042 .880 & 3.369 & 408.260 & 631.251 \\
\hline $\begin{array}{llllllll}\text { Cuenca } & \ldots & \ldots & \ldots & \ldots & \ldots & \ldots & \ldots\end{array}$ & 222.306 & 111.176 & 74.042 & 37.088 \\
\hline $\begin{array}{llllllll}G e r o n a & \ldots & \ldots & \ldots & \ldots & \ldots & \ldots & \ldots\end{array}$ & 441.990 & 125.425 & 135.232 & 181.333 \\
\hline $\begin{array}{lllllll}G r a n a d a & \ldots & \ldots & \ldots & \ldots & \ldots & \ldots\end{array}$ & 736.045 & 99.781 & 267.039 & 369.225 \\
\hline $\begin{array}{llllll}\text { Guadalajara } & \ldots & \ldots & \ldots & \ldots & \ldots\end{array}$ & 139.524 & 68.810 & 25.552 & 45.162 \\
\hline $\begin{array}{lllllll}\text { Guipúzcoa } & \ldots & \ldots & \ldots & \ldots & \ldots & \ldots\end{array}$ & 682.517 & 29.707 & 93.976 & 558.834 \\
\hline $\begin{array}{llllllll}\text { Huelva } & \ldots & \ldots & \ldots & \ldots & \ldots & \ldots & \ldots\end{array}$ & 400.104 & 29.988 & 182.083 & 188.033 \\
\hline $\begin{array}{lllllllll}\text { Huesca } & \ldots & \ldots & \ldots & \ldots & \ldots & \ldots & \ldots\end{array}$ & 216.345 & 89.366 & 39.726 & 87.253 \\
\hline $\begin{array}{lllllllll} \\
\text { Jaén } & \ldots & \ldots & \ldots & \ldots & \ldots & \ldots & \ldots & \ldots\end{array}$ & 645.524 & 23.597 & 335.105 & 286.822 \\
\hline 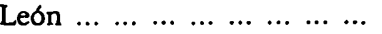 & 526.496 & 160.579 & 159.396 & 206.521 \\
\hline $\begin{array}{lllllllll}L \text { Lérida } & \ldots & \ldots & \ldots & \ldots & \ldots & \ldots & \ldots\end{array}$ & 349.233 & 119.769 & 104.041 & 125.423 \\
\hline $\begin{array}{lllllll}\text { Logroño } & \ldots & \ldots & \ldots & \ldots & \ldots & \ldots\end{array}$ & 240.736 & 59.198 & 57.629 & 123.909 \\
\hline $\begin{array}{llllllll}\text { Lugo } & (2) & \ldots & \ldots & \ldots & \ldots & \ldots & \ldots\end{array}$ & 390.062 & 9.025 & 241.294 & 139.743 \\
\hline $\begin{array}{lllllll}\text { Madrid (3) } & \ldots & \ldots & \ldots & \ldots & \ldots & \ldots\end{array}$ & 1.092 .676 & 21.127 & 139.711 & 931.838 \\
\hline Málaga ... .. & 919.251 & 43.794 & 152.084 & 723.373 \\
\hline 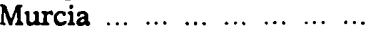 & 884.073 & 7.018 & 103.611 & 773.444 \\
\hline
\end{tabular}




\begin{tabular}{|c|c|c|c|c|}
\hline \multirow[b]{2}{*}{ Provincias } & \multicolumn{4}{|c|}{ Población de los Municipios } \\
\hline & Total & $\begin{array}{l}\text { De hasta } \\
2.000 \text { hab. }\end{array}$ & $\begin{array}{l}\text { De } 2.001 \\
\text { a } 10.000\end{array}$ & $\begin{array}{l}\text { Mayores } \\
\text { de } 10.000\end{array}$ \\
\hline Navarra & 483.867 & 103.130 & 166.459 & 214.278 \\
\hline Orense (4) & 404.945 & 17.075 & 307.822 & 80.048 \\
\hline Oviedo .... & 1.099 .418 & 21.846 & 164.121 & 913.451 \\
\hline Palencia & 186.710 & 72.999 & 50.154 & 63.557 \\
\hline Palmas (Las) & 707.330 & 5.416 & 94.797 & 607.117 \\
\hline Pontevedra ... & 825.607 & 641 & 228.698 & 596.268 \\
\hline Salamanca ....... & 349.843 & 159.898 & 27.004 & 162.941 \\
\hline Santa Cruz de Tenerife & 686.958 & 7.537 & 151.107 & 528.314 \\
\hline $\begin{array}{lllllll} & \\
\text { Santander } & \ldots & \ldots & \ldots & \ldots & \ldots & \ldots\end{array}$ & 490.249 & 57.714 & 141.376 & 291.159 \\
\hline 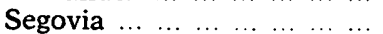 & 151.620 & 73.041 & 30.878 & 47.701 \\
\hline Sevilla .... & 1.375 .540 & 8.145 & 299.826 & 1.067 .569 \\
\hline 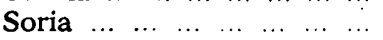 & 103.908 & 52.610 & 22.990 & 28.308 \\
\hline $\begin{array}{cccccc}\text { Tarragona } & \ldots & \ldots & \ldots & \ldots & \ldots\end{array}$ & 484.583 & 83.787 & 123.618 & 277.178 \\
\hline $\begin{array}{llllllll}\text { Teruel } & \ldots & \ldots & \ldots & \ldots & \ldots & \ldots & \ldots\end{array}$ & 155.449 & 85.174 & 35.148 & 35.127 \\
\hline $\begin{array}{llllll} & \ldots & \ldots & \ldots & \ldots & \ldots\end{array}$ & 464.226 & 120.147 & 235.741 & 108.338 \\
\hline Valencia & 1.939 .488 & 139.969 & 335.763 & 1.463 .756 \\
\hline Valladolid & 450.670 & 97.710 & 48.160 & 304.800 \\
\hline $\begin{array}{lllllll}\text { Vizcaya } & (5) & \ldots & \ldots & \ldots & \ldots & \ldots\end{array}$ & 1.150 .593 & 43.586 & 121.413 & 985.594 \\
\hline $\begin{array}{llllllll}\text { Zamora } & \ldots & \ldots & \ldots & \ldots & \ldots & \ldots & \ldots\end{array}$ & 230.787 & 148.914 & 18.109 & 63.764 \\
\hline $\begin{array}{lllll}\ldots & \ldots & \ldots & \ldots & \ldots \\
\ldots\end{array}$ & 802.031 & 128.235 & 89.843 & 583.953 \\
\hline $\begin{array}{llllll}\ldots & \ldots & \ldots & \ldots & \ldots \\
\end{array}$ & 67.077 & - & - & 67.077 \\
\hline $\begin{array}{cccccccc}\text { Melilla } & \ldots & \ldots & \ldots & \ldots & \ldots & \ldots & \ldots\end{array}$ & 59.616 & - & - & 59.616 \\
\hline
\end{tabular}

\begin{tabular}{|c|c|c|c|}
\hline \multirow[b]{2}{*}{ Provincias } & \multicolumn{3}{|c|}{ Población de los Municipios } \\
\hline & $\begin{array}{l}\text { De hasta } \\
2.000 \text { habit. }\end{array}$ & $\begin{array}{l}\text { De } 2.001 \\
\text { a } 10.000\end{array}$ & $\begin{array}{l}\text { Mayores } \\
\text { de } 10.000\end{array}$ \\
\hline & \multicolumn{3}{|c|}{ Porcentajes } \\
\hline $\begin{array}{llllll}\ldots & \ldots & \ldots & \ldots & \ldots & \ldots\end{array}$ & 14,1 & 6,2 & 79,7 \\
\hline 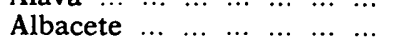 & $\begin{array}{l}14,1 \\
12,3\end{array}$ & 35,1 & 52,6 \\
\hline $\begin{array}{lllllll}\text { Alicante } & \ldots & \ldots & \ldots & \ldots & \ldots & \ldots \\
\end{array}$ & 5,1 & 17,5 & 77,4 \\
\hline $\left.\begin{array}{llllllll}\text { Almería } & \ldots & \ldots & \ldots & \ldots & \ldots & \ldots & \ldots\end{array}\right]$ & 14,2 & 27,8 & 58,0 \\
\hline 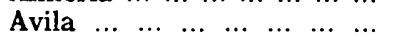 & 53,7 & 28,0 & 18,3 \\
\hline $\begin{array}{lllllll}\text { Badajoz } & \ldots & \ldots & \ldots & \ldots & \ldots & \ldots \\
\end{array}$ & 13,0 & 45,1 & 41,9 \\
\hline $\begin{array}{llllllll}\text { Baleares } & \ldots & \ldots & \ldots & \ldots & \ldots & \ldots\end{array}$ & 2,0 & 73,7 & 24,3 \\
\hline $\begin{array}{cccccccc}\text { Barcelona } & \ldots & \ldots & \ldots & \ldots & \ldots & \ldots \\
\end{array}$ & 4,2 & 15,6 & 80,2 \\
\hline $\begin{array}{lllllllll}\text { Burgos } & \ldots & \ldots & \ldots & \ldots & \ldots & \ldots & \ldots\end{array}$ & 34,6 & 10,4 & 55,0 \\
\hline $\begin{array}{llllllll}\text { Cáceres } & \ldots & \ldots & \ldots & \ldots & \ldots & \ldots & \ldots\end{array}$ & 35,0 & 39,3 & 25,7 \\
\hline $\begin{array}{llllllll}\text { Cádiz } & \ldots & \ldots & \ldots & \ldots & \ldots & \ldots & \ldots\end{array} \ldots$ & 0,3 & 8,9 & 90,8 \\
\hline $\begin{array}{lllllll}\text { Castellon } & \ldots & \ldots & \ldots & \ldots & \ldots & \ldots\end{array}$ & 16,8 & 17,8 & 65,4 \\
\hline $\begin{array}{llllll}\text { Ciudad Real } & \ldots & \ldots & \ldots & \ldots & \ldots\end{array}$ & 10,0 & 35,6 & 54,4 \\
\hline $\begin{array}{lllllll}\text { Córdoba } & \ldots & \ldots & \ldots & \ldots & \ldots & \ldots\end{array}$ & 2,9 & 29.6 & 67,5 \\
\hline $\begin{array}{llllll}\text { Coruña }(\mathrm{La}) & \ldots & \ldots & \ldots & \ldots & \ldots\end{array}$ & 0,3 & 39,2 & 60,5 \\
\hline 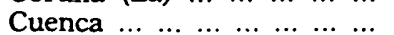 & 50,0 & 33,3 & 16,7 \\
\hline 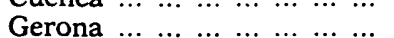 & 28,4 & 30,6 & 41,0 \\
\hline $\begin{array}{lllllll}\text { Granada } & \ldots & \ldots & \ldots & \ldots & \ldots & \ldots\end{array}$ & 13,6 & 36,3 & 50,1 \\
\hline $\begin{array}{cccccc}\text { Guadalajara } & \ldots & \ldots & \ldots & \ldots & \ldots\end{array}$ & 49,3 & 18,3 & 32,4 \\
\hline 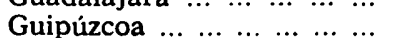 & 4,4 & 13,7 & 81,9 \\
\hline $\begin{array}{lllllllll}\text { Huelva } & \ldots & \ldots & \ldots & \ldots & \ldots & \ldots & \ldots\end{array}$ & 7,5 & 45,5 & 47,0 \\
\hline
\end{tabular}


ESTADISTICA

Población de los Municipios

\begin{tabular}{|c|c|c|c|}
\hline Provincias & $\begin{array}{l}\text { De hasta } \\
2.000 \text { habit. }\end{array}$ & $\begin{array}{l}\text { De } 2.001 \\
\text { a } 10.000\end{array}$ & $\begin{array}{l}\text { Mayores } \\
\text { de } 10.000\end{array}$ \\
\hline & & Porcentajes & \\
\hline $\begin{array}{llllllll}\text { Huesca } & \ldots & \ldots & \ldots & \ldots & \ldots & \ldots & \ldots\end{array}$ & 41,3 & 18,4 & 40,3 \\
\hline $\begin{array}{lllllllllll}\text { Jaén } & \ldots & \ldots & \ldots & \ldots & \ldots & \ldots & \ldots & \ldots\end{array}$ & 3,7 & 51,9 & 44,4 \\
\hline $\begin{array}{cllllllll}\text { León } & \ldots & \ldots & \ldots & \ldots & \ldots & \ldots & \ldots & \ldots\end{array}$ & 30,5 & 30,3 & 39,2 \\
\hline $\begin{array}{llllllll}\text { Lérida } & \ldots & \ldots & \ldots & \ldots & \ldots & \ldots & \ldots\end{array}$ & 34,3 & 29,8 & 35,9 \\
\hline $\begin{array}{lllllll}\text { Logroño } & \ldots & \ldots & \ldots & \ldots & \ldots & \ldots\end{array}$ & 24,6 & 23,9 & 51,5 \\
\hline $\begin{array}{lllllllll}\text { Lugo } & \ldots & \ldots & \ldots & \ldots & \ldots & \ldots & \ldots & \ldots\end{array}$ & 2,3 & 61,9 & 35,8 \\
\hline $\begin{array}{lllllll}\text { Madrid } & \ldots & \ldots & \ldots & \ldots & \ldots & \ldots\end{array}$ & 1,9 & 12,8 & 85,3 \\
\hline $\begin{array}{ccccccc}\text { Málaga } & \ldots & \ldots & \ldots & \ldots & \ldots & \ldots\end{array}$ & 4,8 & 16,5 & 78,7 \\
\hline $\begin{array}{llllllll}\text { Murcia } & \ldots & \ldots & \ldots & \ldots & \ldots & \ldots & \ldots\end{array}$ & 0,8 & 11,7 & 87,5 \\
\hline $\begin{array}{llllllll}\text { Navarra } & \ldots & \ldots & \ldots & \ldots & \ldots & \ldots & \ldots\end{array}$ & 21,3 & 34,4 & 44,3 \\
\hline $\begin{array}{llllllll}\text { Orense } & \ldots & \ldots & \ldots & \ldots & \ldots & \ldots & \ldots\end{array}$ & 4,2 & 76,0 & 19,8 \\
\hline $\begin{array}{lllllllll}\text { Oviedo } & \ldots & \ldots & \ldots & \ldots & \ldots & \ldots & \ldots\end{array}$ & 2,0 & 14,9 & 83,1 \\
\hline $\begin{array}{llllllll}\text { Palencia } & \ldots & \ldots & \ldots & \ldots & \ldots & \ldots & \ldots\end{array}$ & 39,1 & 26,9 & 34,0 \\
\hline $\begin{array}{llllll}\text { Palmas }(\text { Las) } & \ldots & \ldots & \ldots & \ldots & \ldots\end{array}$ & 0,8 & 13,4 & 85,8 \\
\hline $\begin{array}{lllllll} & \text { Pontevedra } & \ldots & \ldots & \ldots & \ldots & \ldots\end{array}$ & 0,1 & 27,7 & 72,2 \\
\hline $\begin{array}{llllllll}\text { Salamanca } & \ldots & \ldots & \ldots & \ldots & \ldots & \ldots\end{array}$ & 45,7 & 7,7 & 46,6 \\
\hline Santa Cruz de Tenerife ... & 1,1 & 22,0 & 76,9 \\
\hline $\begin{array}{llllllll}\text { Santander } & \ldots & \ldots & \ldots & \ldots & \ldots & \ldots\end{array}$ & 11,8 & 28,8 & 59,4 \\
\hline $\begin{array}{llllllll}\text { Segovia } & \ldots & \ldots & \ldots & \ldots & \ldots & \ldots & \ldots\end{array}$ & 48,2 & 20,3 & 31,6 \\
\hline $\begin{array}{cccccccc}\text { Sevilla } & \ldots & \ldots & \ldots & \ldots & \ldots & \ldots & \ldots\end{array}$ & 0,6 & 21,8 & 77,6 \\
\hline $\begin{array}{lllllllll}\text { Soria } & \ldots & \ldots & \ldots & \ldots & \ldots & \ldots & \ldots\end{array}$ & 50,6 & 22,1 & 27,3 \\
\hline $\begin{array}{ccccccc}\text { Tarragona } & \ldots & \ldots & \ldots & \ldots & \ldots & \ldots\end{array}$ & 17,3 & 25,5 & 57,2 \\
\hline $\begin{array}{lllllllll}\text { Teruel } & \ldots & \ldots & \ldots & \ldots & \ldots & \ldots & \ldots\end{array}$ & 54,8 & 22,6 & 22,6 \\
\hline $\begin{array}{cccccccc}\text { Toledo } & \ldots & \ldots & \ldots & \ldots & \ldots & \ldots & \ldots\end{array}$ & 25,9 & 50,8 & 23,3 \\
\hline $\begin{array}{lllllll}\text { Valencia } & \ldots & \ldots & \ldots & \ldots & \ldots & \ldots\end{array}$ & 7,2 & 17,3 & 75,5 \\
\hline $\begin{array}{llllllll}\text { Valladolid } & \ldots & \ldots & \ldots & \ldots & \ldots & \ldots\end{array}$ & 21,7 & 10,7 & 67,6 \\
\hline $\begin{array}{llllllll}\operatorname{Vizcaya} & \ldots & \ldots & \ldots & \ldots & \ldots & \ldots & \ldots\end{array}$ & 3,8 & 10,6 & 85,6 \\
\hline $\begin{array}{llllllll}\text { Zamora } & \ldots & \ldots & \ldots & \ldots & \ldots & \ldots & \ldots\end{array}$ & 64,5 & 7,9 & 27,6 \\
\hline $\begin{array}{ccccccc}\text { Zaragoza } & \ldots & \ldots & \ldots & \ldots & \ldots & \ldots\end{array}$ & 16,0 & 11,2 & 72,8 \\
\hline
\end{tabular}

Los Municipios con más de 10.000 habitantes, abstracción hecha, una vez más, de Madrid y Barcelona, absorben el 65 por 100 de la población española; pero su distribución por Provincias es dispar. Se acentúa esta concentración urbana en 19 Provincias, cuya distribución geográfica es muy similar a la expuesta en el apartado III en relación con las Provincias con tasas de incremento de población, en el quinquenio, superiores a la media nacional, con la salvedad de que el número de Provincias afectadas es más limitado en el litoral mediterráneo -Barcelona, Castellón, Valencia, Alicante y Murcia-, que se extiende en Andalucía, además de Cádiz, a Sevilla, Málaga y Córdoba, a las que hay que añadir Zaragoza.

El predominio de la población rural se presenta en un mayor número de Provincias, 28, con tasas superiores al promedio nacional. Constituyen una gran zona, sin solución de continuidad, que 
comprende todas las Provincias de la Meseta, a excepción de Madrid y Ciudad Real, todas las del Valle del Ebro; Gerona, Tarragona y Castellón, en el litoral mediterráneo, y Santander al Norte.

Cuatro de ellas - Alava, Castellón, Valladolid y Zaragoza - tienen también un valor preponderante en Municipios de zona urbana.

En la zona intermedia, 24 Provincias presentan valores superiores al nacional. Tan sólo Baleares, Ciudad Real, La Coruña, Huelva y Jaén, Lugo y Orense resaltan solamente en la zona intermedia. La mayor parte de las demás coinciden con Provincias en las que predomina la población rural, a excepción de Córdoba y Pontevedra, con predominio de población urbana.

Constituye esta Crónica un avance informativo sobre la población municipal en 1975, en espera de los resultados de la explotación estadística que nos permita conocer sus características más significativas en el orden personal, familiar y social. 
REVL-1977, núm. 193. BALLESTER ROS, IGNACIO. LA EVOLUCION DE LA POBLACION MUNICI...

REVISTA

DE

ESTUDIOS

(*) DE IA

- VIDA LOCAL

\section{JURISPRUDENCIA}


REVL-1977, núm. 193. BALLESTER ROS, IGNACIO. LA EVOLUCION DE LA POBLACION MUNICI...

REVL-1977, núm. 193. BALLESTER ROS, IGNACIO. LA EVOLUCION DE LA POBLACION MUNICI... 